\title{
Trends on primary TKA in Government Tertiary-Referral Hospitals 2019
}

\author{
Reza Rahman Ramadhani ${ }^{1}$, Dicky Mulyadi², Armia Indra Nur Alam³ ${ }^{3}$, Cokorda Gde Oka Dharmayuda ${ }^{4}$ \\ Sholahuddin Rhatomy ${ }^{5}$, Rizki Rahmadian ${ }^{6}$, Muhammad Andry Usman ${ }^{7}$, Chairiandi Siregar ${ }^{8}$, Asep Santoso ${ }^{9}$, \\ Krisna Yuarno Phatama ${ }^{10}$
}

Investigation performed at The Department of Orthopaedic and Traumatology, Faculty of Medicine, Brawijaya University-Saiful Anwar General Hospital, Malang

\begin{abstract}
Background: Total knee arthroplasty (TKA) is the most common surgical option for end-stage knee OA with knee deformity and persistent pain. This procedure has already been increased in national insurance era. There are many conditions may affect the outcome of TKA include: the complexity or difficulty of the surgery or specific states of the affected limb. The difficult conditions in primary TKA are extra-articular deformity, severe coronal deformity, stiffness knee, neglected dislocation or patella subluxation, post-osteotomy (HTO or DFO), previous incision surgery. The paper aims to review the conditions which may adversely affect the usually excellent outcome of TKA
\end{abstract}

Methods: This is a descriptive study. The study describes the difficult or complex situation in primary TKA. The data for this study was obtained from the 9 Government Hospital in Indonesia. The patients receive surgery between January and December 2019.

Results: Totally, there were 881 patients receive TKA in 9 government hospitals in Indonesia during 2019. From those, there were 221 difficult TKA cases 31,38\%, and 600 were the simple case $(68,11 \%)$. Severe coronal deformity are 183 cases. TKA with the stiff knee are 52 cases. Cases with wound problems or previous incision are 12 cases. Ekstra articular deformity are 15 cases. TKA post osteotomy are 9 cases. TKA with genu recurvatum are 6 case. TKA with patella dislocation or subluxation is 4 case

Conclusions: Almost one-third of primary TKA are difficult cases that need much more advanced technique and advance implant (more expensive) for more extended durability (avoid failure and costly-revision). National insurance coverage should recognize primary-difficult TKA

Keywords: Total Knee Arthroplasty, Primary, Government hospital

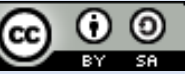

This is an open-access article under the CC-BY-SA license.

\section{Article history}

Submitted: May $30^{\text {th }}, 2020$

Revise : July $23^{\text {th }}, 2020$

Accepted : July $24^{\text {th }}, 2020$
Corresponding Author: Krisna Yuarno Phatama, MD, Department of Orthopaedic and Traumatology, Faculty of Medicine, Brawijaya University-Saiful Anwar General Hospital, Jalan Jaksa Agung Suprapto No. 2, Klojen, Malang, 65112, East Java, Indonesia Email of Krisna Yuarno Phatama:

krisnayuarno@ub.ac.id 


\section{Introduction}

Total Knee arthroplasty (TKA) is the most common surgical option for later stage OA with knee abnormalities and persistent pain. This procedure has been increased in the National Insurance era. TKA has demonstrated excellent results on long-term follow-up with small levels of complications in many series over the last three decades. However, there are many conditions that can affect the outcome of the TKA due to the complexity or difficulty of the operation or particular state of the affected limbs. The difficult conditions in TKA include: extra-articular deformity, severe coronal deformity, stiff knee, neglected dislocations or subluxations of patella, post osteotomy (HTO or DFO), previous incision operations, which carries a greater risk level causing the threshold to perform operations may be higher than for the main routine of TKA. Proper surgical techniques and adequate instrumentation are essential for enhancing functional outcomes. But National insurance does not recognize and differentiate between the difficult cases and the simple cases of primary TKA. This paper aims to review conditions that could have a bad impact on the remarkable results of either TKA.

\section{Methods}

Study and sample design

This research is descriptive. The data for this study came from 9 hospital government centers in Indonesia. The patients were candidates undergoing surgery between January and December 2019. The 881 patients are registered according to the following inclusion criteria. The inclusive criteria are patients undergoing primary TKA, patients undergoing TKA with previous osteotomy surgery, patients undergoing TKA with severe coronal deformity, patients undergoing TKA with extra-articular deformity, patients undergoing TKA with previous incision, stiff knees, patients undergoing TKA with patella dislocation or subluxation. Patients underwent major TKA in 9 tertiary referral government hospitals.

\section{Extra-articular deformity}

An extra-articular deformity located beyond the attachment of a collateral ligament, thereby involving a proximal to the femur epicondyle or tibia distal to the head fibular. Extra-articular deformities can be uni-, bi-, or tri-planar, depends on whether they are isolated or joint defects in coronal (varus-valgus), sagital (flexion-extension), or transverse (rotational) and on whether they are associated with intra-articular instability. We determined on the basis of a pre-operative line of a long film heavy bearing radiograph.

\section{Previous surgical incision}

Previous incisions may appear after procedures such as open reduction and internal fixation (ORIF) for fractures, ligament reconstruction, open and osteotomy. The skin should be evaluated for previous fistulae or infection sites, and skin adhesion to the bones

\section{Severe coronal deformity}

Severe coronal form deformity can be defined as one involving a fixed varus or valgus deviation $\geq 20^{\circ}$ of a neutral mechanical axis on a heavy-bearing film

\section{A recurvatum genu}

Osteoarthritis of the knee with genu recurvatum or hyperextension $>5^{\circ}$ may be secondary to a variety of conditions

\section{Post osteotomy}

The criteria of these patients are those with high osteotomy tibia or distal femoral osteotomy. Indications for the following TKA osteotomy are secondary pain for arthrosis, joint stiffness, recurrence of deformity, progression to tri-compartmental arthrosis.

\section{TKA with Patella dislocation or subluxations}

TKA with Patella dislocation or subluxations is commonly associated with genu valgus, external rotation, hypoplasia of the lateral femoral condyle or patella.

\section{Stiff knees}

Knee stiffness sings rigid knee selection criteria (a pre-operative bow of 50 or less motion can involve a limited flexion or extension or combined. 


\section{Results}

This is a descriptive research. A Total of 881 patients experienced TKR at 9 government hospitals in Indonesia. There are 221 difficult cases at TKA 31, 38\% and 600 is a simple case $(68,11 \%)$. TKA with stiff knees is 52. The previous wound or incision problem was 12. Extraarticular deformities are 15 cases. Post an osteotomy TKA is 9 cases. A severe coronal deformity are 183 cases. The TKA with genu recurvatum is 6 cases. TKA with patella dislocation or subluxations is 4 cases. The most common difficult case is TKA with severe coronal abnormalities with 183 cases is true. The most common cases are severe coronal form deformity. The second and third most common difficult cases are stiff knees and extra-articular deformity. The less common difficult case in TKA is TKA with Patella dislocation or subluxations

Table 1. Total amount simple \& difficult case primary TKA

\begin{tabular}{|l|l|l|}
\hline Difficult case primary TKA & 281 & $31,89 \%$ \\
\hline Simple case primary TKA & 600 & $68,11 \%$ \\
\hline Total & 881 & $100 \%$ \\
\hline
\end{tabular}

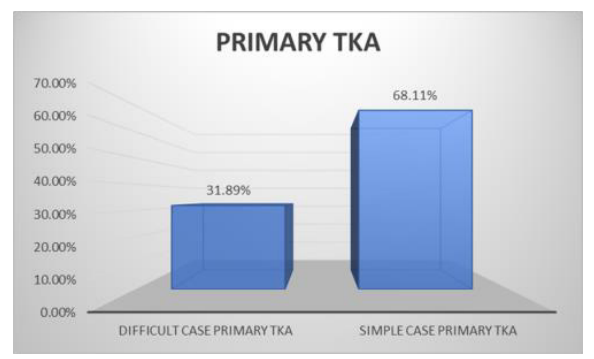

Graphic 1. Simple \& difficult case primary TKA

Table 2. Distribution Difficult case in primary TKA

\begin{tabular}{|l|l|l|}
\hline \multicolumn{3}{|l|}{ Difficult Case Primary TKA } \\
\hline Severe Coronal Deformity & 183 & $65 \%$ \\
\hline Stiff Knee & 52 & $18.51 \%$ \\
\hline Ekstraarticular Deformity & 15 & $5.34 \%$ \\
\hline Previous wound & 12 & $4.27 \%$ \\
\hline Post osteotomy & 9 & $3.20 \%$ \\
\hline Genu Recurvatum & 6 & $2.13 \%$ \\
\hline Dislocation/subluxation Patela & 4 & $1.42 \%$ \\
\hline Total & 281 & $100 \%$ \\
\hline & & \\
\hline
\end{tabular}

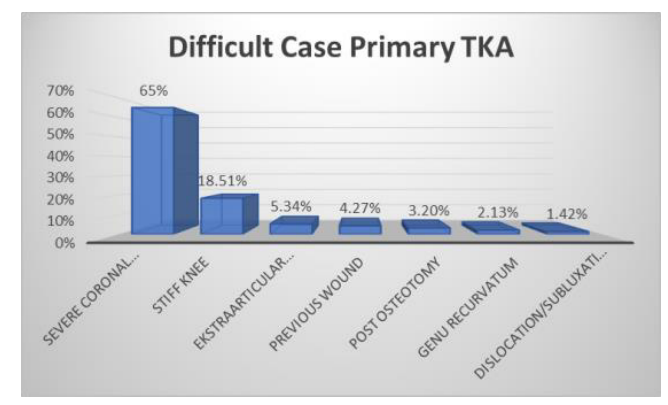

Graphic 3. Distribution Difficult case primary TKA

\section{Discussion}

The purpose of this descriptive study is to describe complexity or difficult cases for primary TKA. From the results of $31,89 \%$ or nearly one primary case is a difficult case. The most common difficult cases in primary TKA are the severe coronal deformity, stiff knees, and extra-articular deformity. Complexity or difficult case in primary TKA carries a greater risk level of complications causing a threshold to perform operations may be higher than for the main routine of TKA. Therefore, from this study we can make predictive and preparation to overcome the difficulties of TKA. An important element to the success of complex problem management is the comprehensive pre-operative planning, including a thorough history and physical examinations, precise techniques and proper instrumentation. In this study we explained the techniques of addition and instrumentation more advanced than the simple primary TKA.

\section{Severe coronal deformity(varus or valgus $\geq 20^{\circ}$ )}

In this situation, we have a standard bone the tibialis resection to prevent a larger gap, causing more thickness of the Polyethylene insert or constraint. The tibial cutting jig faces the lateral tibial spine in to match the tibial anatomical axis in severe varus deformity. But in severe valgus deformity, the proximal positioning of the tibial jig cutting is medial relative to the metaphyseal center and calculate at pre-operative planning radiograph. The osteophyte must be resected first before do some relese in extension and flexion first.

In varus deformity,for tight extension gap, we release semimembranous tendon posterior medial capsule, posterior oblique ligament, deep MCL gradually from inside to outside. For tight flexion gap, we ensure femoral AP rotation cutting \& tibia cutting correctly, lateralization of tibial component, medial tibial condyle 
reduction osteotomy, downsizing of tibial component, release anterior fiber superficial MCL.

In severe valgus deformity, for tight ekstension gap, it's need to release ITB, LCL, posterolateral capsule from outside to inside or release $\mathrm{PCL}$, posterolateralcapsule, ITB from inside to outside (Ranawat technique). For tight flexion gap, it's need to ensure femoral AP rotation cutting \& tibia cutting are correctly, if necessary release popliteus tendon. It must be considered cement, metal augments or bone graft depending on the severity of bone defects. Consider advancement of $\mathrm{MCL}$ or use a constrain implant if there is still ligament laxity.

\section{Stiff knees}

In operative stage, it must to equal and balance flexion-extension gap trial component \& real component before wound closure, ensure the accurate cutting jigs \& bone resection, alignment, prevent patella baja, overstuff patellofemoral, remove all osteophyte inclucding posterior ostephyte, including release posterior capsule, ensure $\mathrm{PCL}$ not too tight and doesn't limit flexion in Cruciate retaining implant. Fibrous adhesion bands and synovial tissue in the patellofemoral and suprapatellar area were excised. The medial and lateral gutters of the knee were developed fully. Debulking, with excision of the lateral aspect of prepatellar fad pad, and division of the lateral patellofemoral ligaments helped in patellar eversion. The quadriceps contracture poses a problem during surgical, it need to proceeding with a $v-y$ quadricepsplasty, snip, or tibial tubercle osteotom. We can resect more distal femur for adding extention or more resect posterior condyle for adding flexion

\section{Extra-articular deformity}

Degree abnormalities of the deformity and its location (distance from the joints) should be determined especially if the extra-articular deformities are present. The general rule is closer deformities are to the joints, the greater the impact of deformities on bone resection. It is important to determine whether an intraarticular correction osteotomy will harm the collateral ligament. The plain knee radiograph that includes full Hip-to-ankle film is recommended to decide the operation to address the case. There are 3 types of operations in this situation which are one staged Intraarticura correction, one stagedextraarticular correction/ osteotomy with TKA, two staged extra-articular correction (TKA \& osteotomy). Deformities $>20^{\circ}$ in sagittal plane and $>10^{\circ}$ in coronal plane needs ekstraaricular correction/ osteotomy to reduce implant use and limited augmentation
We determine bone resection based on the mechanical axes and the anatomy of a full weaightbearing radiograph. If the line to resection the bone perpendicular to the mechanical axis of the femur and tibia. If the cutting disrupts the collateral ligament, correction osteotomy or limiting the imlpan is indicated.
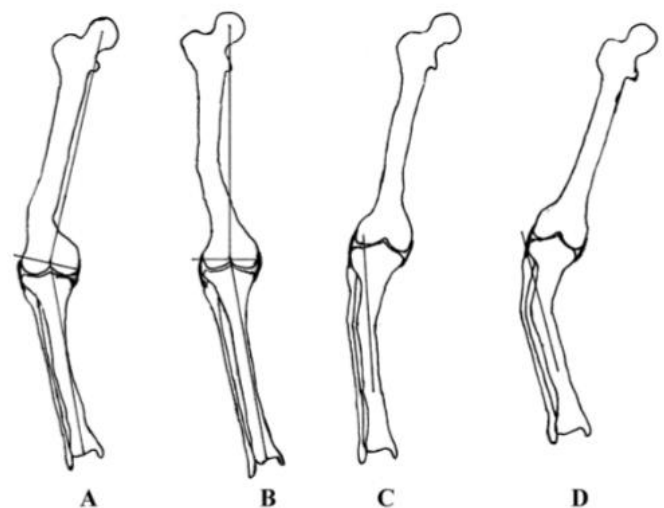

Figure 1. Measurement for ekstraarticular deformity resection

One staged intraarticular correction TKA performed which the deformity $<20^{\circ}$ in sagittal plane and $<10^{\circ}$ in the coronal plane for femur and tibia. It's need ekstramedulary guide or navigation to adjust the bone resection or we adjust the degree of intramedullary femur jig angle and tibia cutting guide base on the measurement bone resection which is must perpendicular with the mechanical axis. Resect and prepare bone graft (If the defect was $>5 \mathrm{~mm}$, a corticocancellous autograft was used), augmentation in large deformity or use PSI (Patient Spesific Instrument) to prevent extensive ligament release which need constrain implant.

One staged extra-articular correction simultaneous corrective femoral osteotomy with TKA, to correct severe mechanical malalignment prior to total knee arthroplasty. It's recommend that osteotomy site be secured with a plate or a locked intramedullary nail or stemmed tibia-femoral component After we correct the alignment according anatomical \& mechanical axis, the arthroplasy will performed with standard bone resection and preserve the ligament balancing. It will minimize the requirement of augmentation \& constrain implant

Two-stage exstra-articular correction have same indication as one-stage extrarticular corection. Stage one is osteotomy correction and fix with plate or intramedullary nail then second staged perform TKA hich one we choose those procedure depend on the patient condition, comorbidities, quality of bone. But there are number of complications associated with corrective 


\section{The previous incision}

In this case we should consider the previous incision, previous fistulas, infections, skin adhesion to the bones, systemic conditions such as smoking, obesity, renal and hepatic insufficiency, the use of corticosteroids and diabetes and scarring, stiffness, which increases the intensity and reduces oxygen tension causes wound damage. To solve this problem are to create a new incision, using the same incision, use the part of the same incision, use the prophylactic flap, make a new medial incision $6 \mathrm{~cm}$ from the previous parallel lateral incision. If there are three or more parallel incision before, the most lateral should be selected. If combined with the stiffness of the knee we can add an length approach (for stiff knees) ex: quadriceps snip, $V-Y$ quadriceplasty, lateral parapatelar approach with tuberositas tibia osteotomy (TTO). Angle of surgical incision and incison before making no more than 60 degree.

\section{Genu recurvatum}

To overcome this difficulties, It's need to cut distal femur less $2 \mathrm{~mm}$ than normal cutting with minimize amount tibia cutting, put smaller size femoral component and the posterior capsule doesn't need to be released to improve the stability, sometimes need augmentation. The bone deformity is greater than $15^{\circ}-20^{\circ}$ need corrective osteotomy or constrain.

For neurological recurvatum case ec polyiomyelitis to prevent hyperextention,it's need constrain/ rotating hinge device and add extention stem to distribute the stress around the stem and decrease the stress to the cement-bone interfaces. It suggested that a constrained prosthesis should be used for elderly patients with a grossly unstable knee and/or bone deformity whether or not they have significant quadriceps power loss and instability

\section{TKA with patella dislocation or subluxation.}

Deformities such as genu valgum and external tibial torsion with OA are commonly associated with this condition. This is especially common with a hypoplastic patella, hypoplastic lateral femoral condyle, and a fiat sulcus. Contracture of the iliotibial band, lateral patellofemoral ligaments, vastus lateralis, and lateral retinaculum prevents the patella from passive reduction. The knee is exposed and a sequential lateral soft tissue release is done to address the valgus deformity. It need ensure the rotation of tibia \& femoral component correctly. A proximal realignment of the patella with an approxımately $\angle \mathrm{cm}$ posterıor to the Iateraı poraer ot the patella and extending superior, separating the vastus lateralis from the rectus femoris tendon. For the medial, the cut edge of the vastus medialis is sutured to the lateral edge of the cut rectus tendon at the level of the superior pole of the patella. A second suture is placed overlapping the medial retinaculum across the lower border of the patella. The amount of overlap is variable depending on the preoperative patellar position. The knee should comfortably flex to $90^{\circ}$ without undue tension on the preliminary sutures. The patella is then resurfaced if it is not too small or eroded to accept a prosthesisfrom. The thickness level patellar cut surface is between 12 and 15 $\mathrm{mm}$. The bone-patellar component should be $1-2 \mathrm{~mm}$ thinner than the original . Install the patellar component medially and superiorly on the cut patellar surface.

\section{Post osteotomy}

There are several problem in TKA post osteotomy. On the post osteotomy TKA, proper alignment and stability can be obtained by routine bone cutting and softtissue balancing course. Hardware removal must be performed staged to anticipate wound healing problems prior to TKA. TKA after the HTO, there is a lateral tendency of articular lines with a higher medial than the bone deficiency of the lateral condyle tibia that may require the use of augmentation or grafting, and the extention of the stem. The posterior slope of the the tibia after the HTO may be neutral, or even reversed. It needs to count the number of tilt resection upside down posterior tibia on the sagittal radiograph. A proximal the tibial metadiaphyseal mismatch after the HTO should be identified in order to predict the possibility of cortical contact with the the Tibialis and the need for offsets. Prepare augmentation, tibia extentian stem, even an exstraarticular osteotomy in case of lateral bone deficiency post HTO

A lateral plate after the DFO can be removed via a long midline incision and a parapatellar medial standard approach if the extensor mechanism has sufficient flexibility. The entry point should be planned where the femoral anatomical axis intersect the lateral condilus femur instead of the intercondylar notch in the post-DFO knee and it's cut must perpendiclair mechanical axis. For this case, TKA after removing internal fixation, we have to put a stem extension. Reasons for using the extension stem generally to bypass the potential stress from metal removal.

\section{Conclusions}

Almost one third or $31,89 \%$ of the primary cases of TKA in 9 government referral hospitals in 2019 is a difficult 
case. Most of them are severe coronal deformities, which may require more advanced techniques and advance implants (more expensive) for more extended durability (avoiding failure and costly-revisions). National insurance coverage must recognize difficult TKA

\section{Conflict of Interest}

The authors affirm no conflict of interest in this study

\section{Acknowledgement}

None

\section{References}

1. Jordan L, Kligman M, Sculco TP. Total knee arthroplasty in patients with poliomyelitis. J Arthroplasty 2007;22:543-548

2. Giori NJ, Lewallen DG. Total knee arthroplasty in limbs affected by poliomyelitis. J Bone Joint Surg [Am] 2002;84-A:1157-1161

3. Berend KR, Lombardi AV Jr, Adams JB. Total knee arthroplasty in patients with greater than 20 degrees flexion contracture. Clin Orthop Relat Res 2006;452:83-87.

4. McAuley JP, Harrer MF, Ammeen D, Engh GA. Outcome of knee arthroplasty in patients with poor preoperative range of motion. Clin Orthop Relat Res 2002;404:203-207.

5. Lonner JH, Siliski JM, Lotke PA. Simultaneous femoral osteotomy and total knee arthroplasty for treatment of osteoarthritis associated with severe extraarticular deformity. J Bone Joint Surg [Am] 2000;82A:342-348.

6. Mullaji A, Shetty GM. Computer-assisted total knee arthroplasty for arthritis with extra-articular deformity. J Arthroplasty 2009;24:1164-1169.

7. van Raaij TM, Reijman M, Furlan AD, Verhaar JA. Total knee arthroplasty after high tibial osteotomy. A systematic review. BMC Musculoskelet Disord 2009;10:88.

8. Hau RC, Newman JH. Knee replacement for osteoarthritis secondary to chronic patellar dislocation and trochlear dysplasia. Knee 2008;15:447-450

9. Saleh KJ, Sherman $P$, Katkin $P$, et al. Total knee arthroplasty after open reduction and internal fixation of fractures of the tibial plateau: a minimum five-year follow-up study. J Bone Joint Surg Am 2001;83-A:1144-1148.

10. Ritter MA, Faris GW, Faris PM, Davis KE. Total knee arthroplasty in patients with angular varus or valgus deformities of $>$ or $=20$ degrees. J Arthroplasty 2004;19:862-866.

11. Ranawat AS, Ranawat CS, Elkus M, et al. Total knee arthroplasty for severe valgus deformity. J Bone Joint Surg [Am] 2005;87-A(Pt 2) (suppl 1):271-284.

12. Mullaji $A B$, Padmanabhan $V$, Jindal $G$. Total knee arthroplasty for profound varus deformity: technique and radiological resu

13. Mullaji A, Marawar S, Sharma A. Correcting varus deformity. J Arthroplasty 2007;22 (suppl 1):15-19.

14. Meding JB, Keating EM, Ritter MA, Faris PM, Berend ME. Total knee replacement in patients with genu recurvatum. Clin Orthop Relat Res 2001;393:244249.

15. Rajgopal A, Ahuja N, Dolai B. Total knee arthroplasty in stiff and ankylosed knees. J Arthroplasty 2005;20:585-590.

16. Nelson $\mathrm{CL}$, Saleh KJ, Kassim RA, et al. Total knee arthroplasty after varus osteotomy of the distal part of the femur. J Bone Joint Surg [Am] 2003;85-A:10621065.

17. Wang JW, Wang CJ. Total knee arthroplasty for arthritis of the knee with extra-articular deformity. J Bone Joint Surg [Am] 2002;84-A:1769-1774.

18. Baldini A, Adravanti $P$. Less invasive TKA: extramedullary femoral reference without navigation. Clin Orthop Relat Res 2008;466:26942700.

19. Catani F, Digennaro V, Ensini A, Leardini A, Giannini S. Navigation-assisted total knee arthroplasty in knees with osteoarthritis due to extra-articular deformity. Knee Surg Sports Traumatol Arthrosc 2012;20:546551

20. Radke S, Radke J. Total knee arthroplasty in combination with a one-stage tibial osteotomy: a technique for correction of a gonarthrosis with a severe ( $>15$ degrees) tibial extra-articular deformity. J Arthroplasty 2002;17:533-537.

21. Erak S, Naudie D, MacDonald SJ, et al. Total knee arthroplasty following medial opening wedge tibial osteotomy: technical issues early clinical radiological results. Knee 2011;18:499-504.

22. Bullek DD, Scuderi GR, Insall JN. Management of the chronic irreducible patellar dislocation in total knee arthroplasty. J Arthroplasty 1996;11:339-345

23. Baldini $A$, Castellani $L$ et al. The difficult primary total knee arthroplasty: a review. J Bone Joint 2015; 97-B (10 Suppl A): 30-9

24. Karachalios, Sarangi $P$ et al. svere varus and valgus deformities treated by Total Knee Arthroplasty. J Bone Joint Surg (Br) 1994; 76-B;938-42

25. Rossi R, Rosso F et al. Total knee arthroplasty in the valgus knee. SICOT 2014: 38:273-283. 


\section{Affiliations:}

1. Dr. Siaful Anwar Hospital, Universitas Brawijaya, Malang, Indonesia

2. Dr. Hasan Sadikin Hospital, Universitas Padjadjaran, Bandung, Indonesia

3. Dr. Zainul Abidin Hospital, Universitas Syah Kuala, Banda Aceh, Indonesia

4. Sanglah Hospital- Universitas Udayana, Bali, Indonesia

5. Dr. Soeradji Tirtonegoro Hospital, Universitas Gadjah Mada, Klaten, Indonesia

6. Dr. M Djamil Hospital, Universitas Andalas, Padang, Indonesia

7. Dr. Wahidin Sudirohusodo Hospital, Universitas Hasanuddin, Makassar, Indonesia

8. Dr. Adam Malik Hospital, Universitas Sumatera Utara, Medan, Indonesia

9. Prof. Dr. R. Soeharso Orthopaedic Hospital, Universitas Sebelas Maret, Surakarta, Indonesia

10. Dr. Siaful Anwar Hospital, Universitas Brawijaya, Malang, Indonesia 